\title{
REGISTROS DE CONSUMO DE REPTILES (SOUAMATA: LACERTILIA Y SERPENTES) POR AVES DE PRESA DIURNAS (AVES: ACCIPITRIFORMES Y CATHARTIFORMES) EN LA PENÍNSULA DE YUCATÁN, MÉXICO
}

\author{
RECORDS OF CONSUMPTION OF REPTILES (SOUAMATA: LACERTILIA Y SERPENTES) BY DIURNAL BIRDS OF PREY \\ (AVES: ACCIPITRIFORMES Y CATHARTIFORMES) IN THE YUCATÁN PENINSULA, MEXICO
}

\author{
Pedro E. Nahuat-Cervera ${ }^{1,2 *}$, J. Rizieri Avilés-Novelo², Ismael Arellano-CiaU ${ }^{3}$, Luis G. Trinchan- \\ GUERRA ${ }^{4}$ \& EYDER JOEL PACAB-COX ${ }^{5}$ \\ ${ }^{1}$ Ekuneil Península de Yucatán. Calle 52 por 89 y 93, colonia Centro, C.P. 97000, Mérida, Yucatán, México. \\ ${ }^{2}$ Campus de Ciencias Biológicas y Agropecuarias, Universidad Autónoma de Yucatán, Km 15.5. carr. Mérida-Xmatkuil, C.P.97315. Mérida, \\ Yucatán, México, \\ ${ }^{3}$ Ichi Tours, calle 38 por 37, número 180, Colonia centro, C.P. 97780. Valladolid, Yucatán, México. \\ ${ }^{4}$ Calle 47, Lote 6, Colonia Leandro Valle, C.P.97144. Mérida, Yucatán, México. \\ ${ }^{5}$ Campus de Ciencias Sociales, Económico-Administrativas y Humanidades, Universidad Autónoma de Yucatán, Gran San Pedro Cholul, C.P. 97305, \\ Mérida. Yucatán, México. \\ *Correspondencia: pedro.nahuat4@gmail.com
}

Abstract.- Reptiles are an important group in food chains, being predators and preys. Birds of prey or raptors are recognized as a group that takes advantage of amphibians and reptiles as a source of food, with some species of raptors with high preference for reptiles. In this study we report eight cases of predation of reptiles by raptors in different geographical locations of the Yucatán Peninsula.

Keywords.- Diet, iguanas, lizards, raptors, snakes.

Resumen.- Los reptiles son un grupo importante en las cadenas alimenticias, desarrollando tanto el papel de depredadores como de presas. Las aves de presa o rapaces están reconocidas como un grupo que aprovecha a los anfibios y reptiles como fuente de alimento, encontrándose algunas especies de rapaces con alta preferencia a este grupo. En este estudio se reportan ocho casos de depredación de reptiles por aves rapaces en diferentes localidades geográficas en la Península de Yucatán.

Palabras clave.- Aves rapaces, dieta, lagartijas, iguanas, serpientes.

Ladepredación tiene un rol fundamentalen muchasinteracciones comunitarias dentro del ecosistema, presentando relación entre el número de depredadores y la velocidad con la que cazan y consumen a la presa, con la fuerza de la interacción (Miller et al., 2006). Estas interacciones tienen influencia en la supervivencia y reproducción, así como en aspectos morfológicos, fisiológicos, evolutivos y etológicos del depredador y la presa (FernándezArhex \& Corley, 2004); sin embargo, las observaciones de los eventos de depredación en campo se consideran poco frecuentes, encontrando estudios enfocados en ciertas especies de aves o reptiles (Poulin et al., 2001).

Los reptiles forman parte importante de la dieta de ciertas especies de aves rapaces, como el Gavilán de Cooper (Accipiter cooperii), la Aguililla aura (Buteo albonotatus), la Aguililla de Swainson (Buteo swainsoni), el Halcón reidor (Herpetotheres cachinnans) y la Aguililla caminera (Rupornis magnirostris), las cuales aprovechan frecuentemente a este grupo como fuente de alimento (Álvarez-del Toro, 1974; Hiraldo et al., 1991, RodríguezEstrella, 2000; Pough et al., 2004; Lazcano et al., 2017), por lo cual este grupo se consideran un componente de las cadenas alimenticias, impactando en la transferencia de energía y nutrientes, y la existencia de ecosistemas saludables (Vitt \& Caldwell, 2009; Luna-Reyes et al., 2013; Valencia-Aguilar et al., 2013).

Aunado al escaso conocimiento de las aves rapaces en el continente americano (Thorstrom, 2000), son escasos los 
registros detallados de los eventos de depredación de reptiles por aves de presa, especialmente en ecosistemas tropicales (Poulin et al., 2001; Miller et al., 2006), por lo que documentar observaciones de esta índole contribuye a generar información relacionada a la historia natural de ambos grupos de vertebrados, útil para comprender mejor diferentes aspectos de su biología e importancia ecológica en el ecosistema (Vitt \& Caldwell, 2009).

En este escrito, se reportan ocho registros ocasionales de consumo de reptiles (Squamata: Lacertilia y Serpentes) por aves rapaces diurnas (Aves: Accipitriformes y Cathartiformes) en diferentes localidades de la Península de Yucatán, México. Las especies de aves y reptiles fotografiadas se identificaron mediante literatura especializada (Lee, 2000; Clark \& Schmitt. 2017) y se tomaron coordenadas geográficas del sitio de la observación, utilizando el sistema geodésico WGS 84, las cuales se encuentran plasmadas en la figura 1. Para los reptiles se siguió la nomenclatura propuesta por González-Sánchez et al. (2017) para la herpetofauna de la Península de Yucatán, mientras que, para las aves, se siguió el listado de las Aves de México de Berlanga-García et al. (2017). Con el fin de una mejor organización, se dividieron las observaciones en dos grupos correspondientes al de las iguanas y lagartijas (Squamata: Lacertilia), y las serpientes (Squamata: Serpentes).

\section{LACERTILIA}

\section{Observación 1}

Iguana negra de cola espinosa (Ctenosaura similis) consumida por Zopilote sabanero (Cathartes burrovianus) y Aguililla cola blanca

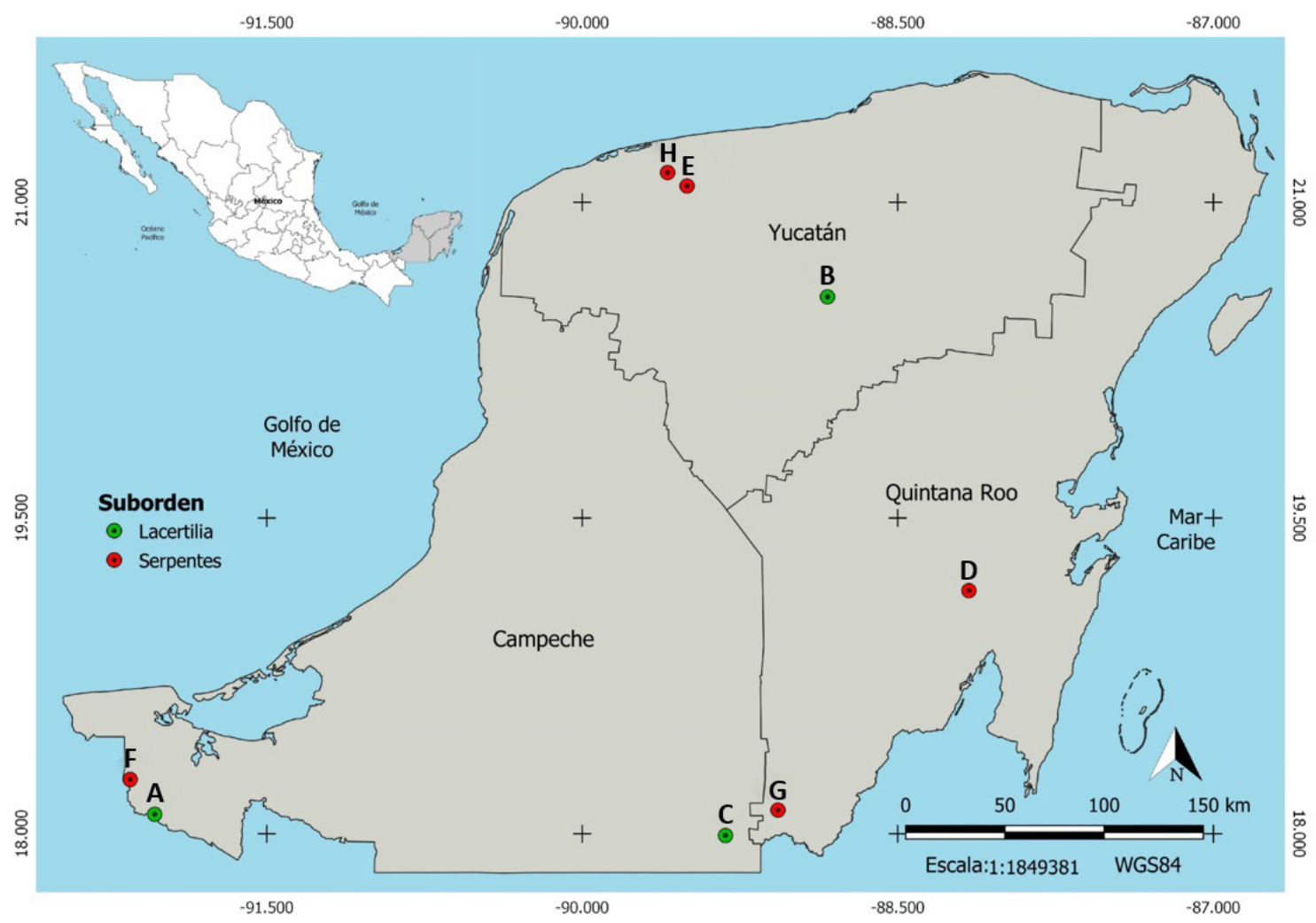

Figure 1. Localities of records records of reptile consumption by diurnal birds of prey in the Yucatan Peninsula, Mexico: A) Cathartes burrovianus and Geranoaetus albicaudatus consuming the body of Ctenosaura similis, B) Buteo albonotatus consuming Laemanctus serratus, C) Buteo plagiatus preying on Holcosus hartwegi, D) Rupornis magnirostris preying on Leptodeira septentrionalis; E) Conophis lineatus; F) Thamnophis proximus; G) Buteogallus urubitinga consuming Leptophis mexicanus; H) Buteo jamaicensis preying on Stenorrhina freminvillii. (Registered letters in the map correspond to the letterss of the next figures).

Figura 1. Localidades con registros de consumo de reptiles por aves de presa diurnas en la Península de Yucatán, México: A) Cathartes burrovianus y Geranoaetus albicaudatus aprovechando el cadáver de Ctenosaura similis, B) Buteo albonotatus consumiendo un Laemanctus serratus, C) Buteo plagiatus depredando un Holcosus hartwegi, D) Rupornis magnirostris depredando a Leptodeira septentrionalis; E) Conophis lineatus; F) Thamnophis proximus; G) Buteogallus urubitinga consumiendo a Leptophis mexicanus; H) Buteo jamaicensis depredando a Stenorrhina freminvillii. (Las letras que están registrados en el mapa corresponden a las letras de las siguientes figuras). 
(Geranoaetus albicaudatus). Municipio de Candelaria, Campeche, México ( $18^{\circ} 05^{\prime} 30.50^{\prime \prime} \mathrm{N}, 92^{\circ} 01^{\prime} 57.50^{\prime \prime} \mathrm{O}$; $\left.06 \mathrm{msnm}\right)$. Potrero con remanentes de vegetación secundaria.

El 6 de marzo de 2019 a 16:20 h se observó el cadáver de un ejemplar adulto de Ctenosaura similis, presuntamente atropellado, sobre la carretera que conecta las comunidades de San Marcos y Palizada, Campeche. El cadáver era devorado por un ejemplar adulto de Cathartes burrovianus. Al acercarnos, el zopilote voló al lado del camino cargando en el pico el cadáver de la iguana para continuar alimentándose del cuerpo. Minutos después llegó un ejemplar adulto de G. albicaudatus, que ahuyentó al zopilote y comenzó a alimentarse del cadáver de la iguana. Posteriormente, el aguililla tomó los restos de la iguana con el pico y voló al interior de la vegetación (Fig. 2A). Ct. similis se identificó por tener un cuerpo robusto, una cola larga con escamas similares a espinas, y una coloración grisácea. C. burrovianus se identificó por su gran tamaño y su plumaje negro, tener una cabeza desnuda de color rojo con tonalidades amarillentas, azules y naranjas, mientras que Geranoaetus albicaudatus posee una coloración gris oscuro en el dorso y la cabeza, mientras que el vientre y el pecho es blanco y las patas amarillas.

\section{Observación 2}

Toloque coronado (Laemanctus serratus) depredado por Aguililla Aura (Buteo albonotatus). Municipio de Yaxcabá, Yucatán, México. $\left(20^{\circ} 33^{\prime} 03.31^{\prime \prime} \mathrm{N}, 88^{\circ} 50^{\prime} 03.97^{\prime \prime} \mathrm{O} ; 30 \mathrm{msnm}\right)$. Vegetación secundaria arbolada con remanentes de selva baja subcaducifolia.

Este evento ocurrió el 28 de abril del 2019 a las 15:47 h. Se escuchó la vocalización de un ejemplar adulto de Buteo albonotatus que posteriormente se observó perchado sobre una rama de un árbol de Guanacaste (Enterolobium cyclocarpum) a una altura aproximada de ocho metros. El ave tenía capturado un ejemplar adulto de Laemanctus serratus, el cual se observó parcialmente consumido. Al notar nuestra presencia, la aguililla detuvo su alimentación, actuó de manera nerviosa y pocos segundos después emprendió el vuelo (Fig. 2B), desapareciendo entre los árboles de los alrededores del sitio de la observación. $L$. serratus se identificó debido a la coloración verde del cuerpo y la cola larga y delgada, características que no presenta otra especie de lagartija de la región, mientras que B. albonotatus presenta patas amarillas, alas largas y delgadas con coloración oscura en las plumas cobertoras y tonalidades más claras en las plumas primarias, secundarias y terciarias, y una cola larga y angosta con bandas blancas.

\section{Observación 3}

Ameiva de Hartweg (Holcosus hartwegi) consumido por Aguililla gris (Buteo plagiatus). Los Tambores de Emiliano Zapata, Municipio de Calakmul, Campeche, México (17 $59^{\prime} 27.15^{\prime \prime}$ $\mathrm{N} ; 89^{\circ} 19^{\prime} 09.97^{\prime \prime} \mathrm{O}$; $163 \mathrm{msnm}$ ). Vegetación de selva mediana subcaducifolia y vegetación secundaria.

El 29 de febrero de 2020 a las 11:45 h se observó a un ejemplar adulto de Buteo plagiatus sobrevolando la carretera que dirige hacia la comunidad de Los Tambores de Emiliano Zapata, en dirección de sur a norte. El ave mantenía apresado con su pico a una lagartija de tamaño mediano (Fig. 2 C), la cual, por la forma de la cabeza, el hocico puntiagudo y el tamaño del cuerpo se identificó como un ejemplar adulto perteneciente al género Holcosus, que por distribución se consideró $H$. hartwegi (MezaLázaro \& Nieto-Montes de Oca, 2015). Esta observación duró escasos segundos ya que el ave se alejó volando de nosotros hacia una zona arbolada al otro lado de la carretera. B. plagiatus se identificó por su coloración gris pálido con un fino patrón barrado en el pecho, mientras que en la cola posee bandas blancas y negras.

\section{SERPENTES}

\section{Observación 1}

Culebra ojo de gato norteña (Leptodeira septentrionalis) consumida por Aguililla caminera (Rupornismagnirostris). Noh Bec, municipio de Felipe Carrillo Puerto, Quintana Roo, México (1909'17.26" N, $88^{\circ} 09^{\prime} 44.68^{\prime \prime} \mathrm{O} ; 25 \mathrm{msnm}$ ). Selva mediana subcaducifolia.

La observación ocurrió el 5 de mayo de 2018 a las $08: 45$ h. Se observó un ejemplar adulto de R. magnirostris posado en el suelo. Al percatarse de nuestra presencia, la rapaz emprendió vuelo hacia un árbol cercano, perchándose en una de sus ramas, en donde se observó que tenía capturada entre las patas un ejemplar adulto de Leptodeira septentrionalis (Fig. 2D). El ave se mantuvo perchada durante unos minutos para posteriormente emprender vuelo, con la serpiente entre sus garras. L. septentrionalis se identificó debido al cuerpo largo y esbelto, cabeza diferenciada del cuerpo por un cuello notorio y patrón de manchas irregulares de coloración oscura, mientras que $R$. magnirostris se identificó por los ojos amarillo claro, patas largas amarillas, y coloración grisácea con estrías café oscuro.

\section{Observación 2}

Culebra guardacaminos (Conophis lineatus) depredada por $R$.

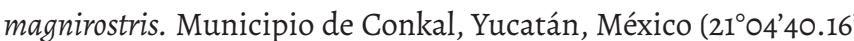


'N; 89 30'07.36" O; $12 \mathrm{msnm}$ ). Vegetación secundaria con remanentes de selva baja caducifolia.

El 19 de septiembre de 2018 a las 08:30 h se observó un ejemplar juvenil de R. magnirostris perchado en las ramas de un árbol seco, a aproximadamente cinco metros de altura. Pocos segundos después de observarlo, este ejemplar voló hacia el suelo y capturó un ejemplar adulto de $C$. lineatus. Al capturar la serpiente, el ave se perchó en un tronco seco a aproximadamente $1.5 \mathrm{~m}$ de altura, donde mantuvo a la serpiente capturada entre sus patas (Fig. 2E). El ave utilizó su pico para tomar la cabeza de la serpiente e iniciar su ingesta. Alrededor de seis minutos después de la captura, el ave devoró completamente a la serpiente. Conophis lineatus se identificó por un cuerpo largo y esbelto, líneas oscuras a los lados de la cabeza y una coloración café claro uniforme en el resto del cuerpo.

\section{Observación 3}

Culebra de agua (Thamnophis proximus) y $R$. magnirostris. Municipio de Palizada, Campeche, México $\left(18^{\circ} 15^{\prime} 26.38^{\prime \prime} \mathrm{N}\right.$; $92^{\circ} 08^{\prime} 57.54^{\prime \prime} \mathrm{O} ; 03 \mathrm{msnm}$ ). Potreros con remanentes de vegetación secundaria y vegetación ribereña.

La observación ocurrió el 16 de febrero de 2019 a las 09:32 h. Se observó a un individuo adulto de $R$. magnirostris perchado en el poste de una cerca, junto a un camino de terracería. El ave tenía entre sus garras a un ejemplar adulto de Thamnophis proximus, el cual había capturado unos minutos antes. La rapaz justo había devoradola cabezadela serpiente, mientras que el restodelcuerpo continuaba moviéndose fuertemente (Fig. 2F). Esta interacción se observó durante aproximadamente 10 minutos, tiempo en el que el ave no se alimentó de la serpiente y se notó nerviosa de nuestra presencia. La serpiente eventualmente redujo la fuerza de sus movimientos, hasta quedarse completamente inmóvil. Se abandonó la escena para evitar perturbar en mayor medida la interacción entre ambas especies. T. proximus se identificó por el cuerpo largo y esbelto, y el patrón conformado por una línea vertebral amarillo claro y líneas amarillo verdoso a los costados.

\section{Observación 4}

Culebra perico mexicana (Leptophis mexicanus) consumida por Aguililla negra mayor (Buteogallus urubitinga). Tres Garantías, municipio de Othón P. Blanco, Quintana Roo, México ( $\left.18^{\circ} 06^{\prime} 42.36^{\prime \prime} \mathrm{N}, 89^{\circ} 04^{\prime} 11.55^{\prime \prime} \mathrm{O} ; 132 \mathrm{msnm}\right)$. Vegetación de tipo selva mediana subcaducifolia.

El 26 de julio de 2019 a las 7:35 h se observó al ejemplar adulto de Buteogallus urubitinga perchado entre las ramas de un árbol de tamaño mediano, a una altura aproximada de $9 \mathrm{~m}$, cerca de un camino de terracería. El ave tenía capturada con sus patas a un ejemplar adulto de Leptophis mexicanus, que realizaba movimientos fuertes para tratar de escapar. Pocos segundos después de la primera observación, el ave ésta tomó con su pico a la serpiente, aún viva, y emprendió el vuelo hacia el otro lado del camino (Fig. 2G), donde había una mayor cantidad de vegetación selvática, Después de esto, no se pudo observar de nuevo a los dos individuos por lo denso de la vegetación. L. mexicanus se identificó por su cuerpo largo y delgado, la coloración dorsal café verdoso y la coloración ventral blanco crema. B. urubitinga se identificó por su tamaño grande, plumaje oscuro, patas largas y amarillas, manchas blancas en los muslos y cola larga con dos bandas blancas.

\section{Observación 5}

Culebra alacranera de sangre (Stenorrhina freminvillii) depredada por Aguililla cola roja (Buteo jamaicensis). Misnebalam, municipio

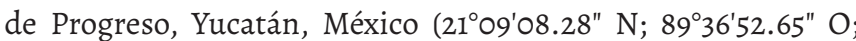
$08 \mathrm{msnm})$. Selva baja caducifolia con parches de vegetación secundaria.

El 05 de diciembre de 2019 a las 10:30 am se observó un ejemplar adulto de Buteo jamaicensis perchada sobre un árbol a una altura aproximada de $6 \mathrm{~m}$ sobre el suelo. Al notar nuestra presencia, el ave emprendió vuelo, evidenciando una serpiente que tenía capturada entre sus patas, la cual se identificó como un ejemplar adulto de Stenorrhina freminvillii (Fig. $2 \mathrm{H}$ ). El ave continuó su vuelo hasta perderse entre la vegetación aledaña al sitio donde se realizó la primera observación. S. freminvillii se identificó por presentar un cuerpo corto y moderadamente robusto, cola corta, una coloración café claro uniforme en el dorso y un vientre blanco crema, mientras que B. jamaicensis presentó la cabeza oscura, el pecho claro con un patrón barrado oscuro, alas anchas y cola con coloración rojiza.

El aprovechamiento de reptiles por parte de las aves rapaces se puede explicar debido a que, en comparación de los mamíferos $y$ las aves, algunos reptiles, como las serpientes, probablemente sean presas más sencillas de capturar, representando un menor desgaste energético para las aves de presa, debido a que en zonas con alta abundancia de roedores se tiene registro de aves de presa cazando serpientes con elevada frecuencia (Selas, 2001). En la Reserva de la Biósfera Michilía, en Durango se registró que alrededor del $20 \%$ de las presas de Buteo albonotatus fueron reptiles, siendo principalmente especies de lagartijas diurnas del género Sceloporus (Hiraldo et al., 1991), mientras que, en Guatemala, se registró que entre el $87.9 \%$ y $100 \%$ de la dieta de Herpetotheres cachinnans se compone de reptiles, con preferencia hacia las serpientes (Parker et al., 2012). 


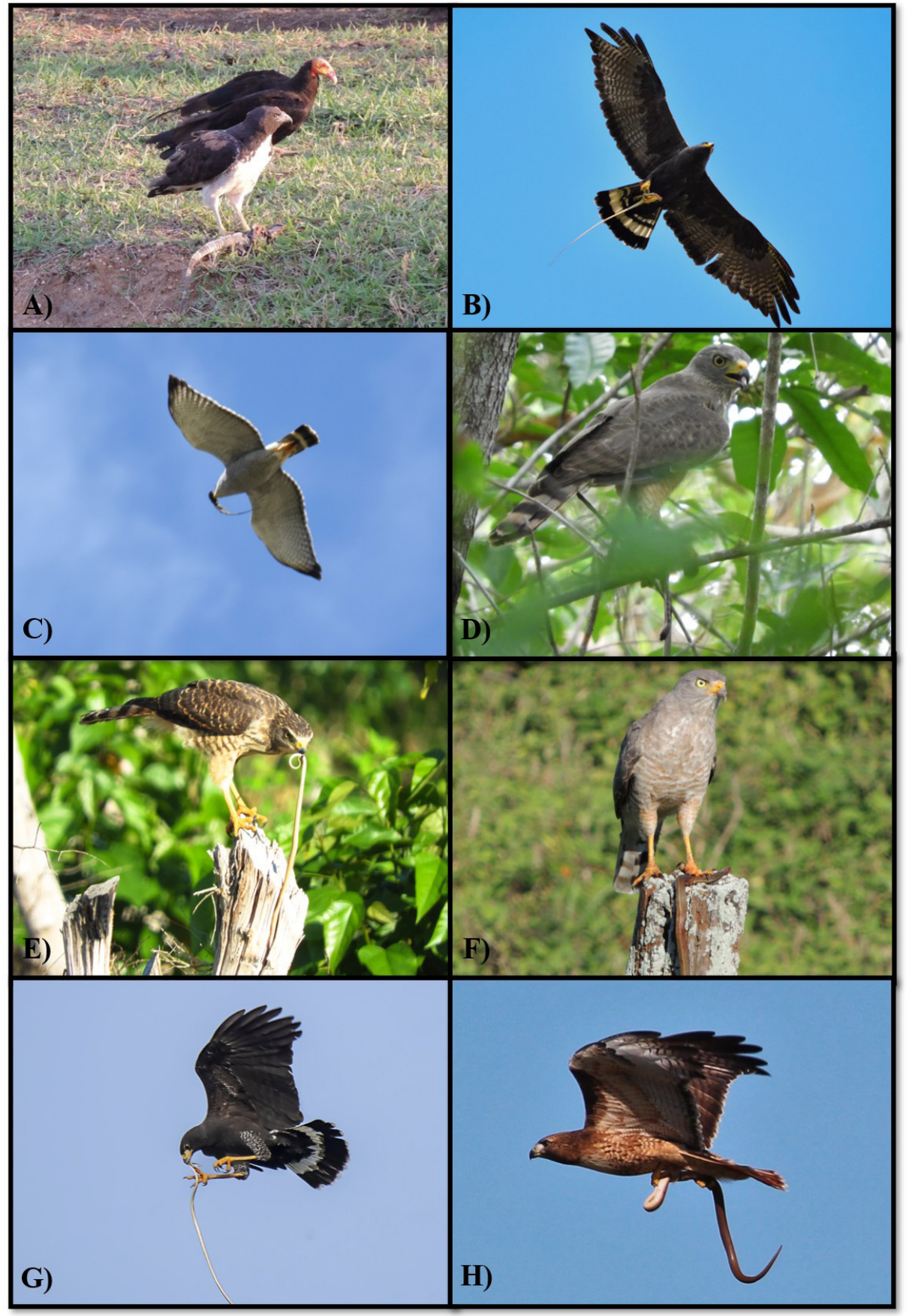

Figure 2. Records of reptile consumptiom by birds of prey in the Yucatan Peninsula: A) Cathartes burrovianus and Geranoaetus albicaudatus consuming the body of an adult specimen of Ctenosaura similis; B) Buteo albonotatus with the partially consumed body of Laemanctus serratus; C) Buteo plagiatus predating an adult specimen of Holcosus hartwegi; D) Rupornis magnirostris preying on Leptodeira septentrionalis; E) Conophis lineatus; F) Thamnophis proximus; G) Buteogallus urubitinga preying on Leptophis mexicanus; H) Buteo jamaicensis preying on adult Stenorrhina freminvillii. Photos by Ismael Arellano-Ciau (A y F), Eyder Joel Pacab-Cox (B), J. Rizieri Avilés Novelo (C), Luis G. Trinchan Guerra (D y E), Rolando Pasos Pérez (G) y Nimish Khanolkar (H).

Figure 2. Registros de consumo de reptiles por aves rapaces en la Península de Yucatán: A) Cathartes burrovianus y Geranoaetus albicaudatus aprovechando el cadáver de un ejemplar adulto de Ctenosaura similis; B) Buteo albonotatus con el cuerpo parcialmente consumido de Laemanctus serratus; C) Buteo plagiatus depredando un ejemplar adulto de Holcosus hartwegi; D) Rupornis magnirostris depredando Leptodeira septentrionalis; E) Conophis lineatus; F) Thamnophis proximus; G) Buteogallus urubitinga depredando a Leptophis mexicanus; H) Buteo jamaicensis depredando un ejemplar adulto de Stenorrhina freminvillii. Fotografías por Ismael Arellano-Ciau (A y F), Eyder Joel Pacab-Cox (B), J. Rizieri Avilés Novelo (C), Luis G. Trinchan Guerra (D y E), Rolando Pasos Pérez (G) y Nimish Khanolkar (H). 
Algunas de las especies de aves reportadas en este escrito han sido previamente observadas aprovechando reptiles dentro de su dieta. Buteogallus urubitinga se ha registrado consumiendo lagartijas y serpientes venenosas y no venenosas; Rupornis magnirostris se alimenta frecuentemente de anfibios y reptiles, siendo registrada alimentándose de crías de Crocodylus acutus y C. moreletii; Geranoaetus albicaudatus incluye comúnmente a los reptiles en su dieta; y Cathartes burrovianus se ha observado alimentándose de lagartijas y serpientes, (Álvarez-del Toro, 1974; Clark \& Schmitt, 2017; Aragao et al., 2020; Da Silva Lucas et al., 2020). Pese a esto, es importante documentar estas interacciones depredador-presa para futuros estudios ecológicos enfocados en las especies mencionadas, ya que las observaciones de historia natural obtenidas durante el trabajo de campo pueden ser consideradas un punto de partida para futuros estudios científicos (Greene, 2005).

En nuestro conocimiento, estas observaciones representan las primeras interacciones documentadas entre las especies de depredador-presa mencionadas en este escrito, por lo que contribuyen al conocimiento de la historia natural de ambos grupos de vertebrados y reflejan la importancia de los reptiles en la dieta de las aves de presa y en las cadenas tróficas de los ecosistemas. Estas interacciones aportan información para el planteamiento de futuras investigaciones enfocadas en conocer aspectos biológicos y ecológicos entre los reptiles y las aves de presa en la Península de Yucatán.

Agradecimientos.- Agradecemos a Rodrigo U. López Valdés, Rolando Pasos Pérez, Ramón Souza Perera, Nimish Khanolkar, Enrique Platas Acosta, Paul Martínez-Córdova y Antonio Granados-Castillo por su valiosa ayuda y asistencia durante el trabajo de campo.

\section{LITERATURA CITADA}

Álvarez-del Toro, M. 1974. Los Crocodylia de México. Instituto Mexicano de Recursos Naturales Renovables A.C, México.

Aragao, M., E.L. Da Silva Brito, A.M. Da Silva Neto, D.M. De Mello Mendes \& R. Sobral. 2020. Tropidurus hispidus (Neotropical Lava Lizard). Predation. Natural History Notes. Herpetological Review 51:135-136.

Berlanga-García, H., H. Gómez de Silva, V.M. Vargas-Canales, V. Rodríguez-Contreras, L.A. Sánchez-González, R. Ortega-Álvarez \& R. Calderón-Parra. 2017. Aves de México. Lista actualizada de especies y nombres comunes. Comisión Nacional para el
Conocimiento y Uso de la Biodiversidad, Distrito Federal, México.

Clark, W.S. \& N.J. Schmitt. 2017. Raptors of Mexico and Central America. Princeton University Press, Princeton, New Jersey, USA.

Da Silva Lucas, P., A. Gomes Poubel \& C.R. Ruiz-Miranda. 2020. Micrurus corallinus (Painted Coral Snake). Predation. Natural History Notes. Herpetological Review 51:148-149.

Fernández-Arhex, V. \& J.C. Corley. 2004. La respuesta funcional: una revisión y guía experimental. Ecología Austral 14:83-93.

Greene, H.W. 2005. Organisms in nature as a central focus for biology. Trends in Ecology and Evolution 20:23-27.

Hiraldo, F., M. Delibes \& J. Bustamante. 1991. Overlap in the diets of diurnal raptors breeding at the Michilía Biosphere Reserve, Durango, Mexico. The Journal of Raptor Research 25:25-29.

Lazcano, D., E. Bailón-Cuellar, G. Ruiz-Ayma, R. MercadoHernández, B. Navarro-Velázquez, L.D. Wilson, G.L. Powell \& A.P. Russell. 2017. Texas Horned Lizards (Phrynosoma cornutum) as prey in Swainson's Hawk (Buteo swainsoni) nest sites at La Reserva de la Biósfera de Janos, Chihuahua, México. Mesoamerican Herpetology 4:885-900.

Lee, J.C. 2000 . A field guide to the amphibians and reptiles of the Mayan world, The lowlands of Mexico, Northern Guatemala, and Belize. 1st Edition. Cornell University Press, New York, USA.

Luna-Reyes, R., L. Canseco-Márquez \& E. Hernández-García. 2013. Los reptiles. Pp. 319-328. En A. Cruz Angón, E.D. Melgarejo, F. Camacho Rico \& K.C. Nájera Cordero (Eds.). La biodiversidad en Chiapas: Estudio de estado. Comisión Nacional para el Conocimiento y Uso de la Biodiversidad (CONABIO) y Gobierno del Estado de Chiapas, México.

Meza-Lázaro, R.N. \& A. Nieto-Montes de Oca. 2015. Long forsaken species diversity in the Middle American lizard Holcosus undulatus (Teiidae). Zoological Journal of the Linnean Society 175: 189-210.

Miller, D.A., J.B. Grand, T.F. Fondell \& M. Anthony. 2006. Predator functional response and prey survival: direct and indirect interactions affecting a marked prey population. Journal of Animal Ecology 75:101-110. 
Parker, M.N., A.M. Enamorado \& M. Lima. 2012. Laughing Falcon. Pp. 265-280. En D.F. Whitacre (Eds.). Neotropical Birds of Prey. Biology and Ecology of a Forest Raptor Community. Cornell University Press, New York, USA.

Pough, F.H., R.M. Andrews, J.E. Cadle, M.L. Crump, A.H. Savitzky \& K.D. Wells. 2004. Herpetology. Third Edition. Pearson Prentice Hall, USA.

Poulin, B., G. Lefebvre, R. Ibáñez, C. Jaramillo, C. Hernández \& A. S. Rand. 2001. Avian predation upon lizards and frogs in a neotropical forest understory. Journal of Tropical Ecology 17:2140.

Rodríguez-Estrella, R. 2000. Breeding success, nest-site characteristics, and diet of the Swainson's hawk (Buteo swainsoni) in a stable population in northern México. Canadian Journal of Zoology 78:1052-1059.
Selas, V. 2001. Predation on reptiles and birds by the common buzzard, Buteo buteo, in relation to changes in its main prey, voles. Canadian Journal of Zoology 79:2086-2093.

Thorstrom, R. 2000. The food habits of sympatric forest-falcons during the breeding season in northeastern Guatemala. Journal of Raptor Research 34:196-202

Valencia-Aguilar, A., A.M. Cortés-Gómez \& C.A. Ruiz-Agudelo. 2013. Ecosystem services provided by amphibians and reptiles in Neotropical ecosystems. International Journal of Biodiversity Science, Ecosystem Services and Management 9:257-272.

Vitt, L.J. \& J.P. Caldwell. 2009. Herpetology. An introductory biology of amphibians and reptiles. Third edition. Academic Press, Elsevier Inc., USA. 